\title{
The effect of psychological capital training on psychological burden and emotional self-regulation styles of mothers having children with cerebral palsy
}

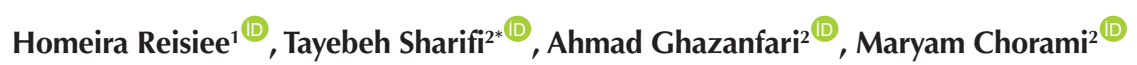 \\ 'Department of Psychology, Shahrekord Branch, Islamic Azad University, Shahrekord, Iran \\ ${ }^{2}$ Shahrekord Branch, Islamic Azad University, Shahrekord, Iran
}

*Corresponding Author: Tayebeh Sharifi, Department of Psychology, Shahrekord Branch, Islamic Azad University, Shahrekord, Iran.Tel: +989131853184, Email: sharifi_ta@yahoo.com

\begin{abstract}
Background and aims: This study aimed to investigate the effect of psychological capital training on psychological burden and emotional self-regulation styles of mothers having children with cerebral palsy.

Methods: This quasi-experimental study was performed in 2019 on mothers of children with cerebral palsy who had files in rehabilitation centers under the supervision of Shahrekord Welfare $(n=270)$. Samples were selected through convenience sampling method such that 30 people were selected from among eligible people and volunteers to cooperate, and then they were randomly divided into experimental and control groups. As for the intervention group, the psychological capital training program was implemented once a week for 10 sessions; but the control group was not given any intervention. Data were collected using the Psychological Burden Questionnaire designed by Zarit et al and the Emotional Self-Regulatory Questionnaire developed by Hoffman and Kashdan in three measurement steps. The collected data were analyzed by multivariate and repeated measure analysis of variance using SPSS-18 software.

Results: The results showed that the mean scores of burden, secrecy, adaptability, and tolerance were significantly different in the post-test compared to the pre-test $(P<0.05)$, as well as in the follow-up stage compared to the pre-test $(P<0.001)$. Furthermore, the mean scores of post-test and follow-up for the experimental group increased in comparison with those for the control group in three styles of emotional regulation, and psychological burden decreased $(P<0.001)$.

Conclusion: The educational intervention of psychological capital could be adopted to increase using adaptive styles of emotional selfregulation, and to reduce psychological burden of mothers having children with cerebral palsy.

Keywords: Psychological capital training, Psychological burden, Emotional self-regulation styles, Cerebral palsy
\end{abstract}

Received: 27 September 2020, Accepted: 7 March 2021, ePublished: December 62021

\section{Introduction}

Cerebral palsy is the most common cause of disability in children, affecting approximately 2.1 per 1000 live births. Traditionally, cerebral palsy is a group of disorders in the development of motor patterns, motor coordination, and posture due to permanent and non-progressive disorders in the developing fetus's brain (1). Families of children with cerebral palsy are out of reach due to their children's constant need for intensive care, frequent medical examinations, as well as ongoing physiotherapy treatments and their role in society (2). Mothers of children with various disabilities suffer from high levels of stress. Children with chronic illnesses cause depression in their mothers emotionally and behaviorally (3).

Primary caregivers of children with physical disabilities often experience a great deal of psychological stress that causes impairment of the individual's emotional functions (4). Emotion regulation includes strategies to increase, maintain, or decrease the intensity, duration, and trajectory of positive and negative emotions (5). The interactive and emotional styles of parents with cerebral palsy can help reduce psychological distress. Parents of children with cerebral palsy experience more stress than parents of children in the whole society, and this parental anxiety and stress is associated with behavioral and behavioral problems in children (6). As the primary caregivers of children with cerebral palsy, mothers experience many social pressures such as anger, shame, and burden (7). Burden of caregiver refers to the negative outcome of the care situation for the caregivers themselves and the person who needs care. In general, caring for a child with a debilitating illness may create high levels of caregiver stress (8). The psychological burden imposed on the caregiver caring for a disabled or mentally ill child can reduce the quality of the provided care and endanger the physical and mental health of the caregiver. Therefore, some therapeutic interventions such as education, support and justified care can have a positive effect on the mental tolerance of the caregivers and improve their quality of life as well as their physical and mental health level (9). 
One of the newest therapies is the psychological capital training which shows the role of positive structures such as hope and optimism in the mental health of vulnerable people (10,11). The psychological capital intervention model is an intervention that emphasizes the growth and development of positive components. This model has been produced and tested by Luthans et al in order to enhance the psychological capital of individuals (12). The four main constructs of psychological capital are selfefficacy (confidence in one's ability and effort to succeed in challenging tasks), hope (perseverance in achieving goals and changing direction towards success if necessary), optimism (being positive about present and future success), and flexibility (including the concept of flexibility in achieving success and goals when facing problems) (13). The dimensions of psychological capital in an interactive and valuable process give meaning to a person's life and help him continue his effort to change stressful situations, get prepared to enter the field of action, and ensure his resilience when facing a difficulty in achieving his goals (14).

Song et al conducted a research review on the given topic and showed that the symptoms of depression pretest, post-test, and follow-up were significantly reduced in the psychological capital intervention group (15). Liu et al have also demonstrated that psychological capital significantly mediates the association of effort/reward and over-commitment with depressive symptoms (16). Tosten and Toprak have revealed that teachers with a high level of positive psychological capital are more successful in cognitive regulation of emotion (17). The results from the studies by Alipor et al have suggested that the implementation of psychological capital intervention increases the mental health of individuals (12).

Considering a number of factors, namely the existence of psychological disorders in mothers whose children have cerebral palsy, the effectiveness of psychological capital training in reducing psychological damage of different people, and the lack of researches examining the effectiveness of psychological capital training in reducing psychological burden and improving emotional self-regulation of mothers having children with cerebral palsy, this study aimed to answer the research question of whether the psychological capital training had an effect on the psychological burden and emotional self-regulation styles of mothers having children with cerebral palsy?

\section{Materials and Methods}

This study is a quasi-experimental single-blinded research with a repeated measure design. The statistical population of the study included mothers with children afflicted with cerebral palsy and having files in rehabilitation centers under the supervision of Shahrekord Welfare Organization in 2020. According to the information obtained from the files, consent forms of participation in the study were sent to 120 eligible families with children aged under 10 years; however, only 69 mothers expressed their voluntary consent to participate in the study. All mothers were given a number. Then, 15 mothers were selected by convenience sampling method, and were randomly divided into intervention group $(n=15)$ and $(n=15)$ control group using a random number table (Figure 1). The sample size was determined to be 15 patients for each group based on the following formula with $95 \%$ confidence interval (18):

$$
\begin{aligned}
& n=\left(\frac{z_{1-\alpha / 2}+z_{1-\beta}}{d}\right)^{2} \\
& z_{1-\alpha / 2}=1.96, z_{1-\beta}=0.84, d=0.72, n=15
\end{aligned}
$$

Inclusion criteria were having a child with cerebral palsy, expressing satisfaction and readiness to participate in the study, not having acute and chronic physical illness according to their health records and counseling in centers, and not having acute and chronic mental illness such as depression and anxiety (according to their health records and counseling). Exclusion criteria, on the other hand, were absenteeism for more than two sessions, lack of cooperation and non-performance of the homework specified in the class, and unwillingness to continue participation in the study. The experimental group received interventions related to psychological capital training in 10 sessions (one session per week) for 90 minutes over two and a half months; but as for the control group, no intervention was made and its members waited to receive the intervention. The description of the psychological capital training sessions was based on the Luthans et al therapeutic-educational protocol (19) in which no interference is made by the researcher in the content of the sessions. A brief description of the sessions is presented in the Table 1.

After the sessions, the follow-up period was carried out two months later to ensure the stability of the results. Mothers participated in the study were assured that their personal information (e.g., demographic characteristics and data from the questionnaire) would remain confidential and the results would be used without mentioning their name and for research purposes only. At the end of the study, in addition, more effective treatment was provided for the people in the control group.

It should be noted that the subjects in the control group who received no intervention as well as the subjects in the experimental group who participated in the training sessions in three stages (before, immediately or two months after the intervention) were given two questionnaires on psychosis and emotion self-regulation.

\section{Psychological burden questionnaire}

This scale was designed in 1980 by Zarit et al to determine the level of mental stress caused by the disease. The questionnaire includes 22 questions about the stress that is imposed on the caregiver as the result of caring for a patient. Scores from never (0) to always (4) are assigned for each question, and the minimum and maximum scores for each person are between 0 and 88 . A higher score indicates more stress, while a lower one suggests less stress; however, 


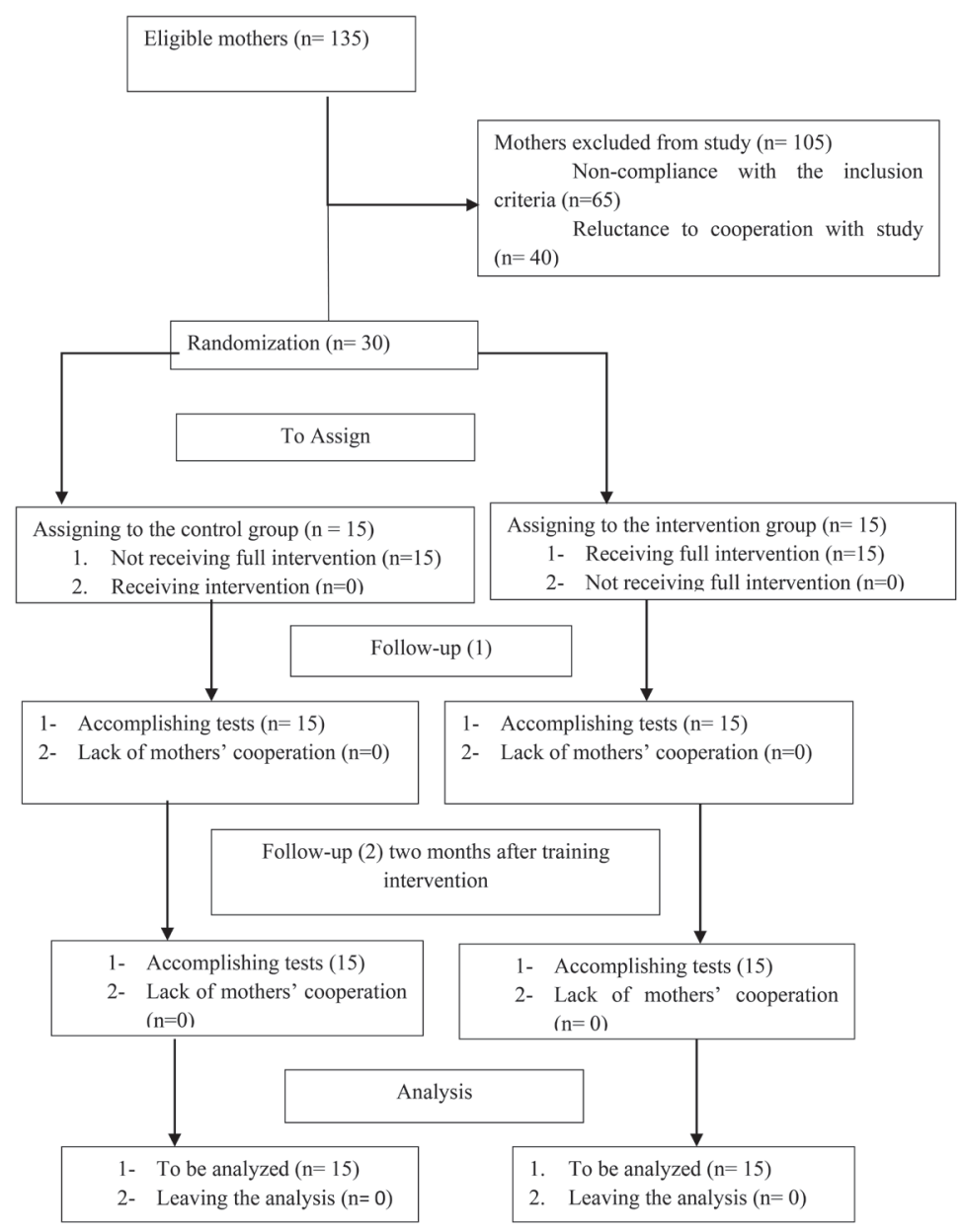

Figure 1. The flowchart of the study procedure

they only provide a general psychological burden score. Zarit et al reported that the reliability coefficient of the original version was 0.71 by retest method, and it was 0.91 by Cronbach's alpha method (20). Navidian and Bahari tested the reliability of this questionnaire using the retest method of 0.94 and its validity in addition to the content analysis method, and its positive and high correlation with Hamilton Anxiety Questionnaire $(r=0.88)$ and Beck Depression Inventory $(r=0.67)$ was confirmed $(21)$.

\section{Emotional Self-Regulation Questionnaire}

This questionnaire was designed by Hoffman and Kashedan in 2010. It includes 20 questions that are answered based on the Likert scale five degrees, from Likert scale (always: 5) to (never: 1). Accordingly, the range of questionnaire's scores is between 20 and 100. A higher score indicates higher emotional self-regulation. This questionnaire has three subscales of adaptability (ability to adjust and regulate emotion), secrecy (emotional inhibition), and tolerance (emotional control in times of stress), which have 8,7 and 5 questions, respectively. In the research study by Kareshki, content validity, principal components analysis, and construct validity of factor analysis were used to determine the validity of the questionnaire. Cronbach's alpha coefficients for the subscales of emotional inhibition, compromise, and tolerance were $0.70,0.75$, and 0.74 , respectively, which demonstrated the acceptable reliability of this scale (22). The reliability of this questionnaire in Najarzadegan and Farhadi research was obtained using Cronbach's alpha coefficient of 0.73 (23).

\section{Statistical analyses}

Collected data were analyzed using descriptive statistics, including frequency, percentage, mean, standard deviation, as well as applying analytical statistics including Fisher's exact tests, multivariate analysis of variance (MANOVA), and repeated measures analysis of variances through SPSS-18. The significance level was considered $P<0.05$.

Results

The majority of subjects in the control group were in the 37-34 age range, academically educated, and housewives. The majority of participants in the intervention group were in the 38-41 age range, academically educated, and housewives. The two groups were almost identical in terms of demographic characteristics $(P>0.05)$ (Table 2$)$. The scores of adaptability, burden, secrecy, and tolerance (dependent variables) of the two groups were compared in 
Table 1. Summary of psychological capital training sessions

\begin{tabular}{|c|c|}
\hline Sessions & Session description \\
\hline 1 & $\begin{array}{l}\text { Introducing the members of the group to each other / expressing the issues, problems, responsibilities and worries of mothers about their children } \\
\text { and examining their coping methods through the transfer of experiences / presenting the group's goals and programs / providing definitions of hope } \\
\text { and despair and characteristics of hopeful people, the concept of optimism, pessimism, / the concept of self-efficacy and the characteristics of self- } \\
\text { sufficient people/ the concept of resilience / the characteristics of resilient people. }\end{array}$ \\
\hline 2 & $\begin{array}{l}\text { Examining the life expectancy and life satisfaction of mothers whose children have cerebral palsy and motivating them, explaining the concept } \\
\text { of learned helplessness and its effect on optimism and pessimism, discussing the role of learned helplessness in reducing self-efficacy, providing } \\
\text { definitions of the concept of toughness and introduce its components (commitment, challenge and control). }\end{array}$ \\
\hline 3 & $\begin{array}{l}\text { Reviewing the previous session and provide a summary of the content in that session, familiarizing mothers with the process of documentation and } \\
\text { the concept of control place, investigating the relationship between motivation, willpower and self-confidence with self-efficacy and using feedback } \\
\text { techniques, focusing on the commitment component and use techniques to improve it. }\end{array}$ \\
\hline 4 & $\begin{array}{l}\text { Goal setting training in personal and family life, teaching how to divide a large goal into smaller goals to increase the likelihood of achieving them, } \\
\text { reviewing and discussing how to increase self-confidence and self-efficacy and providing relevant assignments based on mothers' circumstances } \\
\text { and using positive feedback techniques, focusing on the challenge component, how to turn problems into challenges, and increase your willingness } \\
\text { to face them. }\end{array}$ \\
\hline 5 & $\begin{array}{l}\text { Familiarization of mothers with internal, external, general, specific, stable, unstable documents and the role of each in optimism, using mental } \\
\text { imagery technique to create positive experiences and strengthening it to increase self-efficacy, focusing on the control component and discussing } \\
\text { how to reduce anxiety and increasing a sense of control over life. }\end{array}$ \\
\hline 6 & $\begin{array}{l}\text { Getting feedback from mothers about training and home exercises and examining the problems and obstacles in using them / using the technique of } \\
\text { strengthening substitution by providing global and regional examples of people who are in a condition similar to that of these mothers but but are } \\
\text { auto-efficient, familiarity of mothers with problem-solving and emotion-based strategies and their role in increasing resilience. }\end{array}$ \\
\hline 7 & $\begin{array}{l}\text { Teaching how to create and develop positive documents, training to regulate different emotions and control unpleasant emotions, familiarizing } \\
\text { mothers with scientific, practical and direct methods of problem solving and encouraging mothers to use these strategies. }\end{array}$ \\
\hline 8 & $\begin{array}{l}\text { Introducing members to how to use multiple methods to achieve the goal, using the technique of pleasant event analysis and determining the positive } \\
\text { consequences of these events in order to raise the level of optimism, inviting a successful and self-effective person from the community of mothers } \\
\text { whose children have cerebral palsy to use objective models to increase the level of self-efficacy, learning more about emotion-based strategies and } \\
\text { using them in high stress situations. }\end{array}$ \\
\hline 9 & $\begin{array}{l}\text { Making mothers aware of how barriers become challenges to achieving their goals, emphasizing the use of individual and environmental talents } \\
\text { and abilities and its impact on the level of optimism, talking about the role of controlling place in toughness and using positive self-talk techniques } \\
\text { to increase resilience. }\end{array}$ \\
\hline 10 & $\begin{array}{l}\text { tional status of mothers } \\
\text { d related exercises / revie }\end{array}$ \\
\hline
\end{tabular}

the pre-test. Performing the Box's $\mathrm{M}$ test, the assumption of variance homogeneity was met (Box's M test $=20.135$, $\mathrm{F}=1.699, P=0.075)$.

According to the results from multivariate analysis of variance and Pillai's trace test, there was no significant difference between the two groups regarding dependent variable $(P=0.125)$ (Table 2$)$.

The two groups were re-compared in terms of dependent variable's scores in post-test. The Box's $M$ test showed that the assumption of variance homogeneity was met (Box's $\mathrm{M}$ test $=14.361, \mathrm{~F}=1.212, P=0.278$ ).

According to the results from multivariate analysis of variance and Pillai's trace, a significant difference was observed between the two groups regarding at least one of the dependent variables $(P<0.001)$.

The mean of Burden score in the intervention group was significantly lower than that of the control group. The group variable explained $78.9 \%$ of the variance of Burden score $\left(\mathrm{F}_{(1,28)}=104.703, P<0.001, \eta 2=0.789\right)$. It should be noted that the Burden score decreased by 16 points in the post-test compared to pre-test, while it decreased by 1.6 points in the control group.

The mean of adaptability score in the experimental group was significantly higher than that in the control group. The group variable explained $18.0 \%$ of the variance of adaptability score $\left(\mathrm{F}_{(1,28)}=6.147, P=0.019, \mathrm{\eta} 2=0.180\right)$. (Figure 2) It was demonstrated that the adaptability score increased by 4.34 in the post-test compared to the pretest in the experimental group, while it decreased by 0.06 point in the control group.

The mean of secrecy score in the intervention group was significantly higher than that in the control group. The group variable explained $33 \%$ of the variance of secrecy score $\left(\mathrm{F}_{(1,28)}=13.781, P<0.001, \eta 2=0.330\right)$. The results showed that the secrecy score increased by 5.47 points in the post-test compared to the pre-test in the experimental group, while it increased only by 0.04 point in the control group.

The mean of tolerance score in the intervention group was significantly higher than that in the control group. The group variable explained $50.6 \%$ of the variance of tolerance score $\left(\mathrm{F}_{(1,28)}=28.692, P<0.001, \mathrm{\eta} 2=0.506\right)$.

The two groups were compared in terms of dependent variable's scores in the follow-up stage. The M 'Box test showed that the assumption of variance homogeneity was not met (Box's $M$ test $=35.183, \mathrm{~F}=2.969, P<0.001$ ).

According to the results from multivariate analysis of variance and Pillai's Trace test, there was a significant difference between the two groups concerning at least one of the dependent variables of burden score, secrecy score, 
Table 2. Comparing demographic data of participants in experimental and control groups

\begin{tabular}{lcccc}
\hline \multirow{2}{*}{ Demographic data } & & \multicolumn{2}{c}{ Groups } & \multirow{2}{*}{ P value } \\
\cline { 3 - 4 } & & Control & Experimental & \\
\cline { 2 - 3 } & $30-33$ & $3(20.0)$ & $2(13.3)$ & \\
Age, n (\%) & $38-47$ & $4(26.7)$ & $3(20.0)$ & \\
& $42-45$ & $2(13.3)$ & $4(26.7)$ & 0.905 \\
& $46-49$ & $3(20.0)$ & $2(13.3)$ & \\
Education, & High school \&under & $3(20.0)$ & $4(26.7)$ & \\
n (\%) & Diploma & $6(40.0)$ & $4(26.7)$ & 0.914 \\
& Academic & $6(40.0)$ & $7(42.7)$ & \\
Job, n (\%) & Employed & $5(33.3)$ & $4(26.7)$ & \multirow{2}{*}{1.000} \\
& housekeeper & $10(66.7)$ & $11(73.3)$ & \\
\hline
\end{tabular}

adaptability score, and tolerance score $(P<0.001)$.

The mean of Burden score in the intervention group was significantly lower than that in the control group. The group variable explains $86.2 \%$ of the variance of Burden score $\left(\mathrm{F}_{(1,28)}=175.522, P<0.001, \eta^{2}=0.862\right)$. The results showed that Burden score decreased by 21.33 in the followup stage compared to pre-test in the experimental group, while it decreased by 0.53 point in the control group.

The mean of adaptability score in the intervention group was significantly higher than that in the control group. The group variable explained $45.9 \%$ of the variance of adaptability score $\left(\mathrm{F}_{(1,28)}=23.733, P<0.001, \eta^{2}=0.459\right)$. It should be noted that adaptability score increased by 6.67 in the follow-up stage compared to pre-test in the experimental group, while it decreased by 0.4 point in the control group.

The mean of secrecy score in the intervention group was significantly higher than that in the control group. The group variable explained $59.4 \%$ of the variance of Secrecy score $\left(\mathrm{F}_{(1,28)}=40.968, P<0.001, \eta 2=0.594\right)$. It was found that secrecy score increased by 10.27 points in the followup stage compared to pre-test in the experimental group, while it increased only by 0.53 point in the control group.

The mean of tolerance score in the intervention group was significantly higher than that in the control group. The group variable explained $78.6 \%$ of the variance of tolerance score $\left(\mathrm{F}_{(1.28)}=103.143, P<0.001, \mathrm{\eta}^{2}=0.786\right)$. It was demonstrated that tolerance score increased by 6.73 points in the follow-up stage compared to pre-test in the experimental group, while it increased by 0.273 point in the control group (Table 2).

The results from repeated measures analysis of variance showed that the mean scores of burden, secrecy, adaptability, and tolerance were significantly different in the two groups. the results also suggested that the mean scores of these variables were significantly different in the post-test compared to the pre-test $(P<0.05)$, as well as in the follow-up stage compared to the pre-test $(P<0.001)$.

Comparing the mean of the dependent variables during the studied periods in the two groups indicated that there was no significant difference between the groups regarding the pre-test, but there was a significant difference between the groups concerning the mean in each variable in the post-test and follow-up stage (Tables 3 and 4 and Figure 1).

\section{Discussion}

This study aimed to investigate the effectiveness of psychological capital training in reducing psychological burden and improving self-regulatory styles of mothers whose children had cerebral palsy. The results from multivariate analysis of variance showed that there was no significant difference among the mean scores of psychological burden and three emotion regulation styles (Adaptability, Secrecy and Tolerance) in the experimental and control groups before the intervention $(P>0.05)$. Furthermore, the mean scores for the three styles of mothers' emotional regulation in the experimental group increased immediately and/or two months after the intervention $(P<0.001)$, but the psychological burden decreased significantly $(P<0.001)$. In addition, the results from repeated measure analysis of variance showed that there was a significant difference between control and experimental groups in terms of the scores in three measurements of emotion self-regulation styles and psychological burden; and the interaction effect of the group with time was also significant in mothers' burden and self-regulation styles $(P<0.001)$.These findings were consistent with the study results from Song et al (15), Liu et al (16), Tosten \& Toprak (17), and Alipor et al (12) since the researchers had examined and confirmed the effect of psychological capital on the psychological and communicative health of individuals .

Our findings concerning the positive effect of psychological capital training on mothers' psychological burden could be explained by the fact that psychological capital was a combinative variable of positive components having a synergistic property. This variable facilitated the promotion of human capital values (knowledge and skills) and social capital (the network of relationships between them) based on positive components like hope, optimism, resilience and self-efficacy (24). Liao and Liu reported that people with higher psychological capital were more likely to engage in group interactions and social participation, because psychological capital connected people with others and also caused constant interactions (25). It seemed that teaching problem-oriented direct strategies and encouraging members to use them more frequently, focusing on the control component, and discussing how to increase their sense of control over life and facing the challenges of having a disabled child increased the interaction of them with others and facilitated their acceptance of existing problems and their pursuance of problem-based solutions to life problems. The role of resilience training in training sessions was also worthy of mentioning. Resilience helps a person to improve their social, personal, psychological, and emotional functioning despite being exposed to intense stress. As 
the result of this ability, people who are trained to use resilience techniques of psychological capital intervention often return to normal life with a positive emotion after facing stressful situations (26) and can, therefore, take active strategies to improve the quality of their life and reduce their psychological burden by expanding their psychological ability. Moreover, based on the effectiveness of psychological capital training on the dimensions of emotional self-regulation (adaptation, inhibition and tolerance) of mothers, it can be said that people who positively evaluate and interpret events due to high levels of psychological capital experience a higher level of positive emotions. In fact, psychological capital is formed based on a set of positive thoughts and expectations like self-knowledge as a factor, being purposeful, having positive expectations for the present and the future (27). It seemed that mothers having children with cerebral palsy could feel empowered in how they processed their psychological and emotional processing and prevent dysfunctional emotional processing by gaining selfefficacy from the training of psychological capital and, therefore, could tolerate and control emotional stress and experience less tension. In addition, it seemed that familiarity with internal and external documents, the use of mental imagery, and the use of positive self-talk techniques could increase the ability to adjust and regulate emotion (adaptation) and express emotion in appropriate situations.

The present study faced some limitations including

Table 3. Descriptive indices of dependent variables in experimental and control groups

\begin{tabular}{lccc}
\hline Variables & Time & Control & Experimental \\
\hline \multirow{2}{*}{ Burden $($ Mean \pm SD) } & Pre-test & $55.33 \pm 3.06$ & $57.80 \pm 3.95$ \\
& Post-test & $56.93 \pm 3.69$ & $41.80 \pm 4.38$ \\
& Follow-up & $54.80 \pm 4.20$ & $36.47 \pm 3.34$ \\
& Pre-test & $17.93 \pm 3.59$ & $16.53 \pm 1.68$ \\
Adaptability $($ Mean \pm SD) & Post-test & $17.87 \pm 3.66$ & $20.87 \pm 2.92$ \\
& Follow-up & $17.53 \pm 4.00$ & $23.20 \pm 2.08$ \\
& Pre-test & $18.07 \pm 4.06$ & $17.33 \pm 2.23$ \\
Secrecy $($ Mean \pm SD) & Post-test & $18.47 \pm 3.64$ & $22.80 \pm 2.68$ \\
& Follow-up & $18.60 \pm 3.68$ & $27.60 \pm 4.01$ \\
& Pre-test & $11.73 \pm 3.24$ & $12.87 \pm 2.39$ \\
Tolerance $($ Mean \pm SD) & Post-test & $12.20 \pm 2.62$ & $17.13 \pm 2.42$ \\
& Follow-up & $12.00 \pm 2.70$ & $19.60 \pm 1.06$ \\
\hline
\end{tabular}

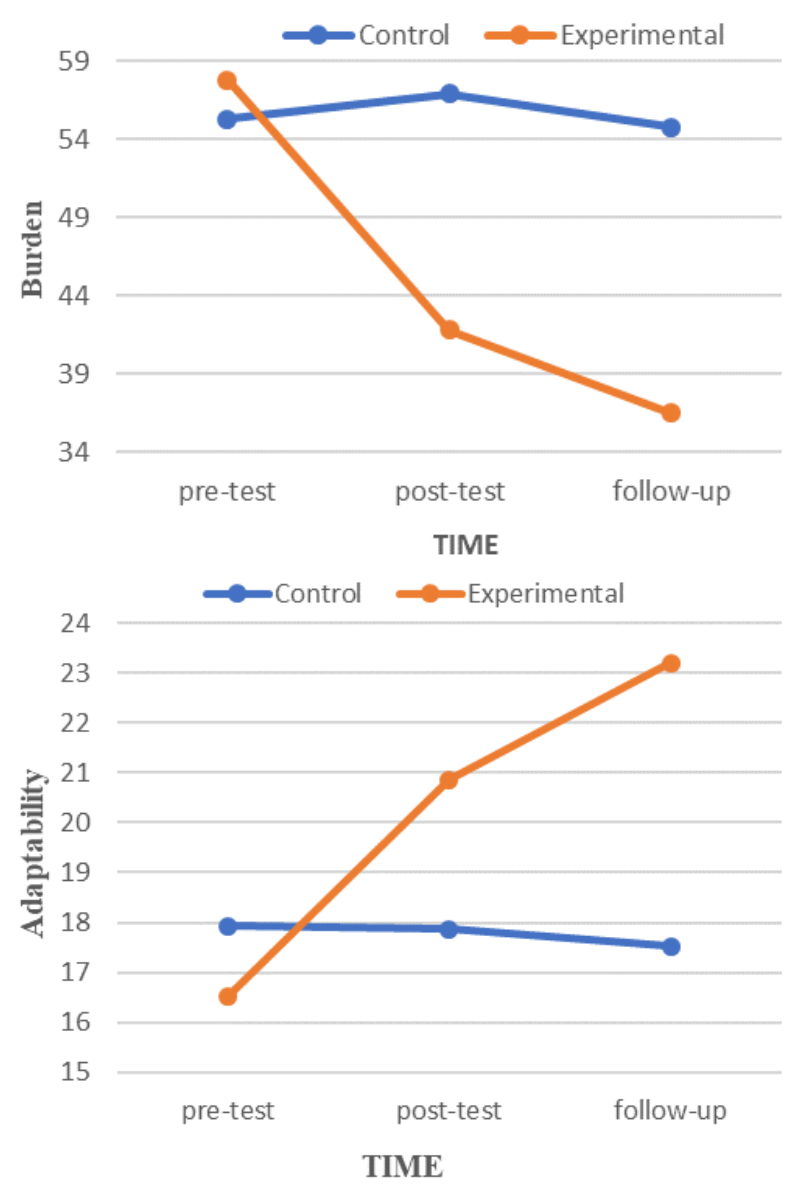

Figure 2 . Mean scores of burdens and adaptability in mothers with cerebral palsy in intervention and control groups.

the narrow scope of the research limited to mothers having children with cerebral palsy in Shahrekord, not using random sampling method, and not controlling the variables (e.g., income, economic and social status, etc) affecting the psychological burden and emotional self-regulation of the participants. Therefore, it was recommended that a study be conducted to examine the fathers having cerebral palsy children as well as mothers having children with other acute and chronic disorders (e.g., thalassemia, cancer) in order for increasing the generalizability of the results. It was also suggested that the impact of economic and social factors as well as the severity of the child's disorder be investigated since they were influential in the psychological burden and emotional distress created in parents.

Table 4. Repeated measures ANOVA results: effects of groups (control, experimental), times (pre-test, post-test, after follow-up), and their interaction groups*times independent variables

\begin{tabular}{|c|c|c|c|c|c|c|c|c|c|}
\hline \multirow{2}{*}{ Independent variables } & \multicolumn{3}{|c|}{ Groups } & \multicolumn{3}{|c|}{ Times } & \multicolumn{3}{|c|}{ Groups*Times } \\
\hline & $\mathbf{F}$ & $d f$ & $P$ & $\mathbf{F}$ & $d f$ & $P$ & $\mathbf{F}$ & $d f$ & $P$ \\
\hline Burden score & 101.62 & 1.28 & 0.001 & 94.472 & 2.56 & 0.001 & 95.92 & 2.56 & 0.001 \\
\hline Adaptability score & 12.30 & 1.28 & 0.002 & 8.406 & 2.56 & 0.001 & 10.44 & 2.56 & 0.001 \\
\hline Secrecy score & 41.84 & 1.28 & 0.001 & 16.681 & 2.56 & 0.001 & 13.52 & 2.56 & 0.001 \\
\hline Tolerance score & 80.95 & 1.28 & 0.001 & 14.847 & 2.56 & 0.001 & 12.29 & 2.56 & 0.001 \\
\hline
\end{tabular}



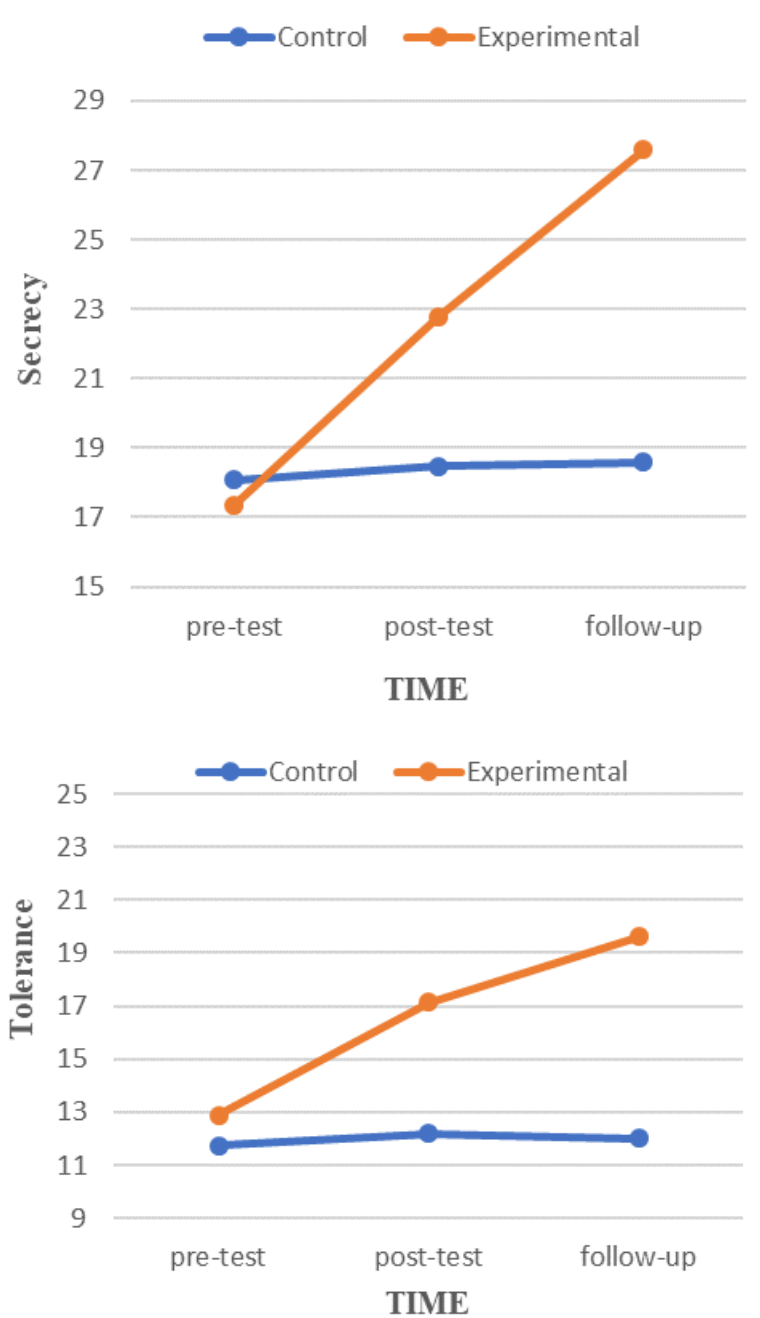

Figure 3. Mean scores of secrecy and tolerance in mothers with cerebral palsy in intervention and control groups.

\section{Conclusion}

It was concluded that the educational intervention of psychological capital could be employed to increase using adaptive styles of emotional self-regulation and to reduce psychological burden of mothers having children with cerebral palsy. Therefore, it was suggested that the psychologists and counselors from health centers for children with cerebral palsy be provided with psychological capital training by setting up specialized workshops. By providing the mothers of these children with this treatment, a practical step is taken in reducing the psychological burden and improving the emotional self-regulation styles of the mothers.

Conflict of Interests

The authors declare that there is no conflict of interests.

\section{Ethical Approval}

Ethical considerations in this study included obtaining permission from the ethics committee of Islamic Azad University Shahrekord Branch (IR.IAU.SHK.REC.1399.012) and obtaining written consent from the participants participating in this study.

Authors' Contributions

$\mathrm{HR}$ and TSH conceived and design the study. AGH supervised intervention sessions and data collection. $\mathrm{HR}$ and $\mathrm{MCH}$ participated in analysis and carried out the data. HR and TSH wrote the first draft of the manuscript. AGH and HR edited the paper. All authors contributed to the writing of the paper, and read and approved the final manuscript.

\section{Funding/Support}

This research received no particular reward from any funding agency in the public, commercial, or not - for - profit sectors.

\section{Acknowledgments}

This article was obtained from a defended PhD thesis in Islamic Azad University of Shahrekord Branch (Approval no: 13320705972019). Hereby, the researchers gratefully thank the participants who willingly participated in this study.

\section{References}

1. Ozkan Y. Child's quality of life and mother's burden in spastic cerebral palsy: a topographical classification perspective. J Int Med Res. 2018;46(8):3131-7. doi: 10.1177/0300060518772758.

2. Pashmdarfard M, Amini M, Shervin Badv R, Ghaffarzade Namazi N, Rassafiani M. Does parent report gross motor function level of cerebral palsy children impact on the quality of life in these children? Iran J Child Neurol. 2017;11(4):52-7. doi: 10.22037/ijcn.v11i4.13545. [Persian].

3. Sigman-Grant M, Hayes J, VanBrackle A, Fiese B. Family resiliency: a neglected perspective in addressing obesity in young children. Child Obes. 2015;11(6):664-73. doi: 10.1089/chi.2014.0107.

4. Tofighi Z, Aghaei A, Golparvar M. Comparing effectiveness of resilience and emotion regulation on perceived social stigma and mental endurance in the mothers of the children with cerebral paralysis (CP). Psychology of Exceptional Individuals. 2018;7(28):71-93. doi: 10.22054/jpe.2018.27210.1670. [Persian].

5. Young KS, Sandman CF, Craske MG. Positive and negative emotion regulation in adolescence: links to anxiety and depression. Brain Sci. 2019;9(4):76. doi: 10.3390/ brainsci9040076.

6. Zamani N, Habibi M, Darvishi M. To compare the effectiveness of dialectical behavior therapy and cognitive behavioral group therapy in reducing depression in mothers of children with disabilities. J Arak Univ Med Sci. 2015;18(1):32-42. [Persian].

7. Del-Pino-Casado R, Rodríguez Cardosa M, López-Martínez C, Orgeta V. The association between subjective caregiver burden and depressive symptoms in carers of older relatives: a systematic review and meta-analysis. PLoS One. 2019;14(5):e0217648. doi: 10.1371/journal.pone.0217648.

8. Schulz R, Sherwood PR. Physical and mental health effects of family caregiving. Am J Nurs. 2008;108(9 Suppl):23-27. doi:10.1097/01.NAJ.0000336406.45248.4c.

9. Emerson E, Brigham P. Health behaviours and mental health status of parents with intellectual disabilities: cross sectional study. Public Health. 2013;127(12):1111-6. doi: 10.1016/j. puhe.2013.10.001.

10. Lamers SM. Positive Mental Health: Measurement, Relevance and Implications [thesis]. Netherlands: University of Twente; 2012.

11. Slade M. Mental illness and well-being: the central importance of positive psychology and recovery approaches. BMC Health Serv Res. 2010;10:26. doi: 10.1186/1472-6963-10-26.

12. Alipor A, Akhondy N, Sarami Foroshany GR, Saffarinia M, Agah Heris M. The effectiveness of psychological capital intervention model $(\mathrm{PCl})$ on the mental health in the experts working in Iran Khodro Diesel co. Iran Occup Health. 2013;10(4):16-24. [Persian].

13. Tang JJ. Psychological capital and entrepreneurship 
sustainability. Front Psychol. 2020;11:866. doi: 10.3389/ fpsyg.2020.00866.

14. Vatankhah Amjad F, Ali Akbari M, Akhoondi N. The effectiveness of cognitive therapy based on mindfulness and psychological well-being of people with chronic pain index referred to sports clubs. The second conference of science and technology of psychology, educational sciences and sociology of Iran, Tehran; 2018. [Persian].

15. Song R, Sun N, Song X. The efficacy of psychological capital intervention $(\mathrm{PCl})$ for depression from the perspective of positive psychology: a pilot study. Front Psychol. 2019;10:1816. doi: 10.3389/fpsyg.2019.01816.

16. Liu L, Chang Y, Fu J, Wang J, Wang L. The mediating role of psychological capital on the association between occupational stress and depressive symptoms among Chinese physicians: a cross-sectional study. BMC Public Health. 2012;12:219. doi: 10.1186/1471-2458-12-219.

17. Tosten R, ToprakM. Positive psychological capital and emotional labor: a study in educational organizations. Cogent Educ. 2017;4(1):1301012. doi: 10.1080/2331186X.2017.1301012.

18. Motamed N, Zamani F. Sample Size in Medical Research: With a Practical Approach. Tehran: Asre Roshan Bini; 2016. [Persian].

19. Luthans F, Youssef CM, Avolio BJ. Psychological Capital: Developing the Human Competitive Edge. Oxford: Oxford University Press; 2007.

20. Zarit SH, Todd PA, Zarit JM. Subjective burden of husbands and wives as caregivers: a longitudinal study. Gerontologist.
1986;26(3):260-6. doi: 10.1093/geront/26.3.260.

21. Navidian A, Bahari F. Burden experienced by family caregivers of patients with mental disorders. Pak J Psychol Res. 2008;23(1-2):19-28.

22. Kareshki H. Evaluating factorial structure of emotional styles in students. J Res Behav Sci. 2013;11(3):185-95. [Persian].

23. Najarzadegan F, Farhadi H. The effectiveness of quality of life therapy on communication skills, addiction to cyberspace, marital satisfaction, and emotional self-regulation in couples. J Psychol Sci. 2019;18(74):247-56. [Persian].

24. Ghashghaeizadeh $\mathrm{N}$. The study of effectiveness of training of psychological capital on dimensions of social capital among women teachers of elementary schools. J Psychol Stud. 2016;12(1):125-40. doi: 10.22051/psy.2016.2255. [Persian].

25. Liao RX, Liu YH. The impact of structural empowerment and psychological capital on competence among Chinese baccalaureate nursing students: a questionnaire survey. Nurse Educ Today. 2016;36:31-6. doi: 10.1016/j.nedt.2015.07.003.

26. Aliyev R, Karakus M. The Effects of positive psychological capital and negative feelings on students' violence tendency. Procedia Soc Behav Sci. 2015;190:69-76. doi: 10.1016/j. sbspro.2015.04.918.

27. Rastegar A, Seif MH, Abedini Y. Presenting a causal model of relationship between psychological capital and teaching emotions: the mediating role of emotional exhaustion. J Appl Psychol Res. 2017;7(4):51-71. doi: 10.22059/ japr.2017.61080. [Persian]. 